\title{
Resolved-Sideband Cooling of a Levitated Nanoparticle in the Presence of Laser Phase Noise
}

\section{Journal Article}

\section{Author(s):}

Meyer, Nadine; de los Rios Sommer, Andrés; Mestres, Pau; Gieseler, Jan; Jain, Vijay; Novotny, Lukas (D); Quidant, Romain

Publication date:

2019-10-11

Permanent link:

https://doi.org/10.3929/ethz-b-000372046

Rights / license:

In Copyright - Non-Commercial Use Permitted

Originally published in:

Physical Review Letters 123(15), https://doi.org/10.1103/PhysRevLett.123.153601 


\title{
Resolved-Sideband Cooling of a Levitated Nanoparticle in the Presence of Laser Phase Noise
}

\author{
Nadine Meyer, ${ }^{1, *}$ Andrés de los Rios Sommer, ${ }^{1}$ Pau Mestres, ${ }^{1}$ Jan Gieseler, ${ }^{1}$ Vijay Jain, ${ }^{2}$ \\ Lukas Novotny, ${ }^{2}$ and Romain Quidant ${ }^{1,3}$ \\ ${ }^{1}$ ICFO Institut de Ciencies Fotoniques, The Barcelona Institute of Science and Technology, Castelldefels, 08860 Barcelona, Spain \\ ${ }^{2}$ Photonics Laboratory, ETH Zürich, 8093 Zürich, Switzerland \\ ${ }^{3}$ ICREA-Institució Catalana de Recerca i Estudis Avançats, 08010 Barcelona, Spain
}

(Received 5 July 2019; published 8 October 2019)

\begin{abstract}
We investigate the influence of laser phase noise heating on resolved sideband cooling in the context of cooling the center-of-mass motion of a levitated nanoparticle in a high-finesse cavity. Although phase noise heating is not a fundamental physical constraint, the regime where it becomes the main limitation in Levitodynamics has so far been unexplored and hence embodies from this point forward the main obstacle in reaching the motional ground state of levitated mesoscopic objects with resolved sideband cooling. We reach minimal center-of-mass temperatures comparable to $T_{\min }=10 \mathrm{mK}$ at a pressure of $p=3 \times 10^{-7} \mathrm{mbar}$, solely limited by phase noise. Finally we present possible strategies towards motional ground state cooling in the presence of phase noise.
\end{abstract}

DOI: 10.1103/PhysRevLett.123.153601

Among the numerous optomechanical systems, Levitodynamical systems excel with an extreme level of isolation from the environment, rendering $Q$ factors exceeding $10^{8}$ [1]. This makes them an attractive alternative to membranes and nanobeams [2-5] for probing macroscopic quantum phenomena at room temperature [6-9]. In addition, Levitodynamics offers unique possibilities unavailable in conventional clamped systems, including free fall [10], rotation [11-14], and engineered potentials [15]. These unique features make them ideal candidates for enhanced sensing applications [16], out of equilibrium thermodynamics [17], and matter wave interferometry $[18,19]$.

Thus far, the motional ground state (GS) of levitated nanoparticles remains elusive. The lowest phonon occupation of tens of phonons, has been achieved with continuous measurement and active feedback cooling [20-24]. In contrast to these active schemes, passive optomechanical cooling provides a way to cool to the GS without continuous measurement, provided that the cavity linewidth is narrower than the mechanical frequency. This socalled sideband cooling technique was originally developed for atomic systems and in combination with cryogenics it has been used for GS cooling $(n<1)$ in a range of optomechanical systems.

The first Levitodynamics experiments demonstrated 1D sideband cooling [25-27] from room temperature down to $0.3 \mathrm{~K}$ [28]. Here we demonstrate 1D resolved sideband cooling of a levitated nanoparticle reaching temperatures of $T_{\min }=10 \mathrm{mK}$ at a pressure of $p=3 \times 10^{-7}$ mbar, a regime where we will show that phase noise heating is indeed the limiting factor. The phonon occupation of the mechanical oscillator yields $n_{\mathrm{ph}} \approx 2100$, an occupation $125 \times$ less than in previous experiments employing resolved sideband cooling [28] and comparable to minimal temperatures reached in coherent scattering [29-32]. Next to the well-known decoherence due to thermal noise and photon recoil [21], we investigate in detail the influence of frequency noise of the cavity field, also called phase noise, on the phonon occupation. Phase noise decoherence has so far been largely overlooked in Levitodynamics [32] despite being previously observed in other platforms [33,34] where it seriously complicates the creation of low phonon states $[35,36]$.

Understanding the limitations of sideband cooling techniques with actively driven cavities is essential for many protocols to generate entanglement [37,38], nonclassical correlations [39], or achieve coherent quantum control [40]. Controlling the mechanical motion of mesoscopic systems on the single phonon quantum level has been achieved only recently $[41,42]$.

By using an external cavity, the center-of-mass (c.m.) motion of an atom, ion, molecule [43,44], or mesoscopic particle can be controlled and therefore cooled. The presence of a polarizable object inside the cavity induces a position-dependent dispersive change in optical path length, altering the intracavity intensity which then acts back on the particle motion. Coherently driving the cavity with a red (blue) detuned light field enhances (reduces) anti-Stokes scattering versus Stokes scattering, thus cooling (heating) the c.m. motion.

The interaction Hamiltonian for a particle moving along the axis of an optical cavity is $\hat{H}_{\text {int }}=-\hbar g_{0} \hat{a}^{\dagger} \hat{a}\left(\hat{b}+\hat{b}^{\dagger}\right)$ 
$[25,45]$, where $\hat{a}\left(\hat{a}^{\dagger}\right)$ is the photon annihilation (creation) operator and $\hat{b}\left(\hat{b}^{\dagger}\right)$ is the phonon annihilation (creation) operator. The single photon optomechanical coupling strength $g_{0}$ can be enhanced by the driving field as $g^{2}=$ $g_{0}^{2} \hat{a}^{\dagger} \hat{a}=g_{0}^{2} n_{\text {cav }}, n_{\text {cav }}$ being the intracavity photon number. The single photon optomechanical coupling strength is sinusoidally modulated due to the intracavity standing wave and given as

$$
g_{0}=U_{0} \sin (2 k y) k \sqrt{\frac{\hbar}{2 m \Omega_{y}}},
$$

where $U_{0}$ is the resonance frequency shift induced by a particle placed at the center of an empty cavity, with $U_{0}=\omega_{\text {cav }} \alpha /\left(2 \epsilon_{0} V_{\text {cav }}\right) \approx 2 \pi \times 10 \mathrm{kHz}, \omega_{\text {cav }}$ being the cavity resonance frequency, $\alpha=4 \pi \epsilon_{0} r^{3}\left(n_{p}^{2}-1\right) /\left(n_{p}^{2}+2\right)$ the polarizability of the particle with radius $r=118 \pm 6 \mathrm{~nm}$ and refractive index $n_{p}=1.45$. The cavity volume is $V_{\text {cav }}=\pi L_{\text {cav }} w_{\text {cav }}^{2} / 4, \quad L_{\text {cav }}=2.43 \mathrm{~cm}$ the cavity length, $w_{\text {cav }}=64 \mu \mathrm{m}$ the cavity waist, $k=2 \pi / \lambda_{\text {cav }}$ the cavity field wave vector, $\lambda_{\text {cav }}=1064 \mathrm{~nm}$ the cavity wavelength, and $y$ the position of the particle from the center along the cavity axis. The particle mass $m=(4 / 3) \pi r^{3} \rho$ is inferred from the particle density $\rho=2200 \mathrm{~kg} / \mathrm{m}^{3}$, and the particle mechanical frequency $\Omega_{m}$ is obtained from the particle displacement power spectral density (PSD). The optomechanical damping rate is then given by [45]

$$
\Gamma_{\mathrm{opt}}=g_{0}^{2} n_{\mathrm{cav}}\left(\frac{\kappa}{\frac{\kappa^{2}}{4}+\left(\Delta+\Omega_{y}\right)^{2}}-\frac{\kappa}{\frac{\kappa^{2}}{4}+\left(\Delta-\Omega_{y}\right)^{2}}\right),
$$

with the cavity linewidth $\kappa=40 \mathrm{kHz}$ (FWHM). The optomechanical damping rate depends strongly on the position along the cavity axis $y$ through $g_{0}$, the intracavity photon number $n_{\text {cav }}$ and detuning from the cavity resonance $\Delta=\omega_{L}-\omega_{\text {cav }}$. In the resolved sideband regime $\left(\Omega_{m} \gg \kappa\right)$ the maximum cooling rate equals $\Gamma_{\mathrm{opt}}=4 g_{0}^{2} n_{\text {cav }} / \kappa \approx$ $2 \pi \times 2 \mu \mathrm{Hz} n_{\text {cav }}$ at optimal red detuning $\Delta=-\Omega_{m}$, enabling an optomechanical damping rate in the $\mathrm{kHz}$ regime in state-of-the-art cavities.

In addition to the coupling rate to the thermal bath $\Gamma_{m}$, shot noise radiation pressure heating (SNRP) due to the cavity field $\left(\Gamma_{\text {cav }}\right)$ and the trapping field $\left(\Gamma_{t}\right)$ are additional decoherence sources [see Eqs. (C2), (C4), (C5) [46] ]. As shown in Sec. C of the Supplemental Material [46], the additional phonon occupation due to the SNRP of the cavity light field $n_{\text {rad cav }} \ll 1$ [see Eqs. (C1) and (C4) [46] ] does not depend on the intracavity photon number, while the SNRP of the trapping light field acts as an additional thermal bath. The latter causes only a small relative offset and will therefore be neglected in the following. Moreover, heating effects due to classical laser intensity noise show a much smaller heating effect compared to SNRP due to the laser's low relative intensity noise [47] and will therefore also be neglected.

In the regime where the thermal mechanical damping is the main decoherence source, the final phonon occupation of the mechanical oscillator is

$$
n_{\mathrm{ph}}=\frac{\Gamma_{\mathrm{opt}} n_{\min }+\Gamma_{m} n_{\mathrm{th}}}{\Gamma_{\mathrm{opt}}+\Gamma_{m}} \approx n_{\mathrm{min}}+\frac{\Gamma_{m} n_{\mathrm{th}}}{\Gamma_{\mathrm{opt}}}
$$

where $n_{\text {th }}=k_{B} T /\left(\hbar \Omega_{m}\right) \approx 6 \times 10^{7}$ is the initial thermal phonon occupation. We neglect the contribution from the thermal photon occupation of the undriven cavity, since $n_{\text {cav }}=\left(k_{B} T / \hbar \omega_{\text {cav }}\right) \ll 1$ for optical frequencies. $n_{\text {min }}$ puts an ultimate limit on the minimum phonon number for $\Gamma_{\text {opt }} \gg \Gamma_{m}$. As a consequence the GS can only be reached in the resolved sideband regime $\left(\Omega_{m}>\kappa\right)$, where $n_{\text {min }}=\kappa^{2} /\left(4 \Omega_{m}\right)^{2}<1$. The c.m. temperature is then $T_{\text {c.m. }}=n_{\text {ph }} \hbar \Omega_{m} / k_{B}$ (solid lines in Figs. 2-4).

In Fig. 1 the experimental setup is displayed. A silica nanoparticle is levitated in an optical tweezers trap [48]

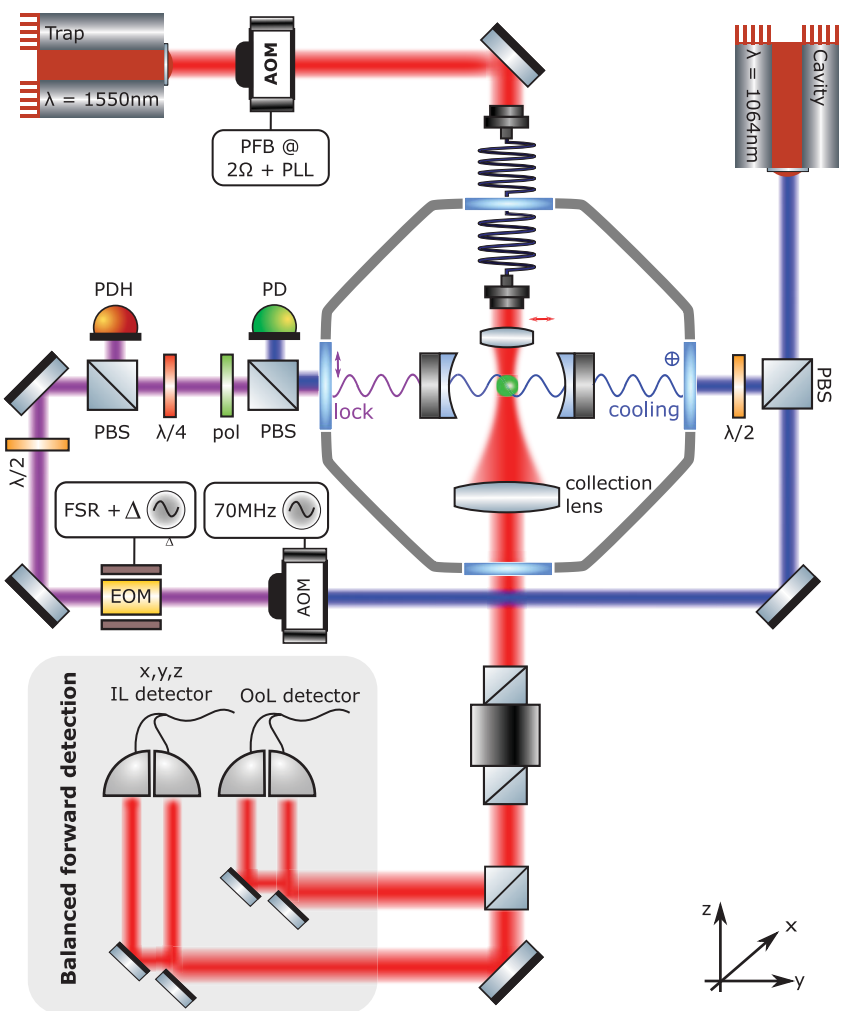

FIG. 1. Experimental setup. The nanoparticle levitates in a mobile optical tweezers trap (red), positioned in the center of the high finesse cavity field. A weak cavity light field (purple) observed on a photodiode (PDH) is used for Pound-Drever-Hall locking on the cavity resonance $\omega_{\text {cav }}$. The cross polarized pump field (blue) is frequency modulated with an EOM at FSR $+\Delta$ and its transmission is recorded (PD). Standard PFC of the optical tweezer trap prevents particle loss and cross coupling between different degrees of freedom $(x, y, z)$. A piezo stage allows for precise $3 \mathrm{D}$ positioning of the particle along the cavity axis. The collected trapping light is used in balanced forward detection. 
with a wavelength $\lambda=1550 \mathrm{~nm}$, power $P \simeq 185 \mathrm{~mW}$, and focusing lens NA $=0.8$. The trap is mounted on a $3 \mathrm{D}$ piezo system allowing for precise $3 \mathrm{D}$ positioning of the particle inside the high finesse Fabry-Pérot cavity with a cavity finesse $F=1.55 \times 10^{5}$ and free spectral range $\mathrm{FSR}=2 \pi \times 6.2 \mathrm{GHz}$ (for more details see Supplemental Material A [46]). Because of tight focusing, the nanoparticle eigenfrequencies $\Omega_{x, y, z}=2 \pi \times(90,100$, and $25 \mathrm{kHz}$ ) are nondegenerate. The maximum single photon optomechanical coupling strength is $g_{0}=2 \pi \times 0.14 \mathrm{~Hz}$, which puts GS cooling seemingly into reach by simply increasing the intracavity photon number to $n_{\text {cav }} \geq$ $4.8 \times 10^{9}$, corresponding to a feasible intracavity power of $P_{\text {intra }}=5.5 \mathrm{~W}$.

In our experiments we vary the cavity input power $P_{\text {in }}$, the detuning $\Delta$ and the position $y$ along the cavity axis in low and high vacuum, respectively. In the following, points represent data and solid lines are theoretical predictions according to Eq. (2). The intracavity photon number, used for theoretical predictions, is calculated from the transmitted cavity power. At low pressure, we apply parametric feedback cooling (PFC) along $x, z$, preventing particle loss and limiting the particle displacement to the linear regime of the optical trap. Experimentally, we deduce $T_{\text {c.m. }}$ from the area of the particle displacement PSD equal to $\left\langle y^{2}\right\rangle$ [49], as shown in Fig. 2(a).

Figure 2(b) shows the pressure dependence of $T_{\mathrm{c} . \mathrm{m} \text {. }}$ at optimal detuning $\Delta=-\Omega_{m}$ and intracavity power of $P_{\text {intra }}=75 \mathrm{~mW}$. At pressures below $p<1 \mathrm{mbar}$, we observe the expected linear decrease of $T_{\mathrm{c} . \mathrm{m}}$. At $T_{\mathrm{c} . \mathrm{m} .} \approx 1 \mathrm{~K}$, cooling becomes ineffective and the temperature levels off
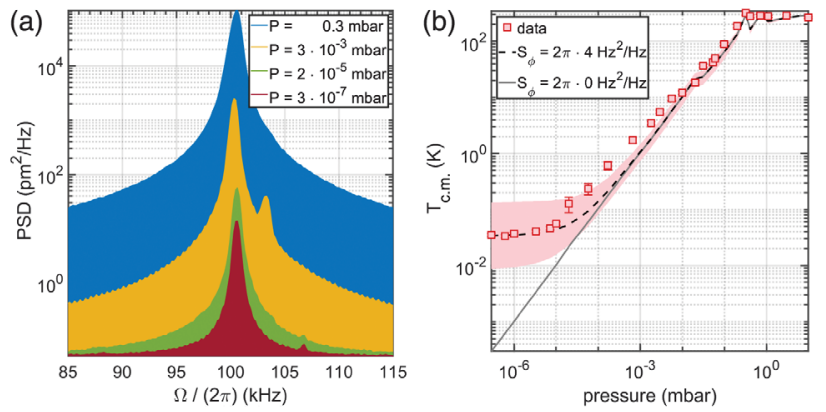

FIG. 2. Temperature versus pressure. (a) PSDs at various pressures $\left(p=0.3,3 \times 10^{-3}, 3 \times 10^{-5}\right.$, and $\left.3 \times 10^{-7} \mathrm{mbar}\right)$. The area of the PSD $\left\langle y^{2}\right\rangle$ and therefore the temperature is reduced by reducing the pressure and applying cavity sideband cooling. (b) Applying cavity sideband cooling at optimal detuning $\Delta=-\Omega_{y}$ and intracavity power of $P_{\text {intra }}=75 \mathrm{~mW} . T_{\text {c.m. }}$. reduces linearly with decreasing pressure down to a stable $T_{\min }=35 \mathrm{mK}$. Theory with negligible phase noise $S_{\phi}=$ $0 \mathrm{~Hz}^{2} / \mathrm{Hz}$ (solid line) predicts a monotonic linear decrease in $T_{\text {c.m. }}$. with pressure. Theoretical predictions assuming phase noise of $S_{\phi}=2 \pi \times 4 \mathrm{~Hz}^{2} / \mathrm{Hz}$ (half-solid line) tails off to a stable final temperature $T_{\text {th }}=34 \mathrm{mK}$. Shaded area assumes a phase noise regime from half to twice the value of $S_{\phi}$. with a constant final minimum temperature of $T_{\min }=$ $35 \mathrm{mK}$, in contrast to theoretical expectations (solid line).

Figures 3 and 4 show measurements at high pressure $p=$ 0.6 mbar (red circle) and low pressure $p=3 \times 10^{-7} \mathrm{mbar}$ (blue square), respectively. In Fig. 3 we investigate $T_{\text {c.m. }}$. versus $\Delta$ for various cavity input powers ranging from $P_{\text {in }}=$ $4-45 \mathrm{~mW}$ at high pressure $(p=0.6 \mathrm{mbar})$ and $P_{\text {in }}=$ $70 \mu \mathrm{W}-4 \mathrm{~mW}$ at low pressure $\left(p=3 \times 10^{-7}\right.$ mbar $)$. At high pressure [Figs. 3(a)-3(c)] $T_{\text {c.m. }}$ features a clear minimum at $\Delta \approx-\Omega_{m}$. The experimental results agree well with the theory, and only for high cavity input powers of $P_{\text {in }}=$ $45 \mathrm{~mW}$ we observe a deviation due to frequency instabilities at high intracavity power. In contrast, at low pressure the data deviate from the theory and the optimal detuning is farther away from resonance as shown in Figs. 3(e)-3(g). Our minimum temperature is $T_{\min } \approx 10 \mathrm{mK}$, corresponding to a minimal phonon number $n_{\min }=2100$. The dependence of $T_{\text {c.m. }}$ at a nominal optimal detuning $\Delta=-\Omega_{m}$ versus cavity input power is summarized in Figs. 3(d) and 3(h) for high and low pressure, respectively. At high pressure $T_{\text {c.m. }}$.
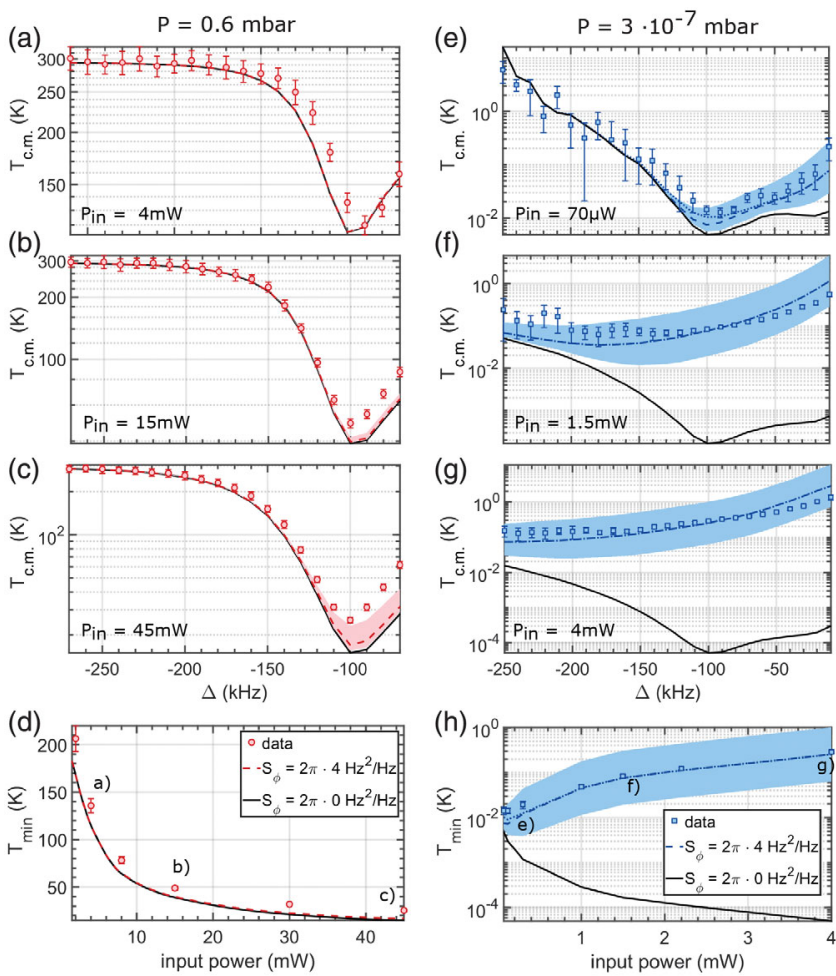

FIG. 3. Temperature versus detuning $\Delta$ for different input powers $P_{\text {in }}$. At high pressures (a)-(d) clear optimal detuning $\Delta_{\text {opt }} \approx-\Omega_{m}$ is observed. At low pressures (e)-(h) the temperature minimum is washed out. Theory assuming $S_{\phi}=0 \mathrm{~Hz}^{2} / \mathrm{Hz}$ (solid line) predicts stronger cooling with increasing $g$. Theory assuming $S_{\phi}=2 \pi \times 4 \mathrm{~Hz}^{2} / \mathrm{Hz}$ (half-solid line) accounts for phase noise. The shaded area assumes a phase noise regime from half to twice the value of $S_{\phi}$. In (d) and (h) $T_{\text {c.m. }}$ at optimal detuning versus $P_{\text {in }}$ is depicted, showing at low pressure the opposite behavior in comparison to high pressure. 

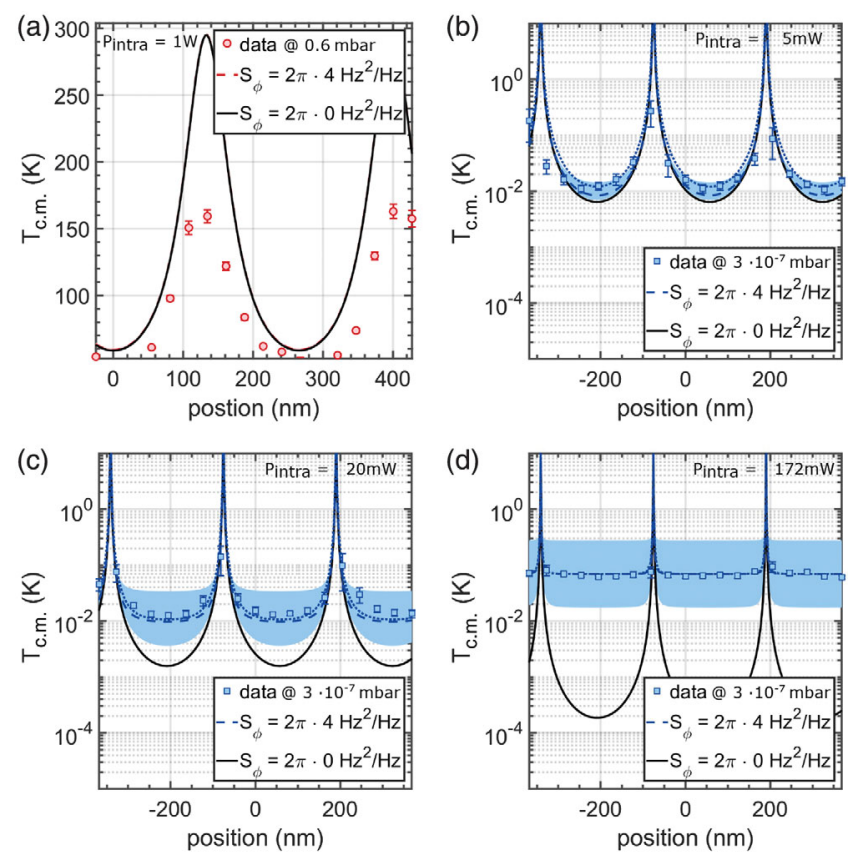

FIG. 4. Temperature versus particle position $y$ at optimal detuning $\Delta=-\Omega_{m}$ and various intracavity powers $P_{\text {intra }}$. (a) At high pressures, $T_{\text {c.m. }}$ changes sinusoidally with position. The minimal temperature of $T_{\min } \approx 50 \mathrm{~K}$ agrees well with theory (solid line). The maximum temperature deviates from the theory prediction due to the movement of the particle in the cavity field, as discussed in the main text. (b)-(d) $T_{\text {c.m. }}$ at low pressures. For the lowest power (b) $T_{\text {c.m. }}$ keeps its sinusoidal dependence on $y$ with a minimal temperature $T_{\min } \approx 10 \mathrm{mK}$. The position dependence is gradually lost and the minimum temperature increases, when the intracavity power is raised. Theory neglecting phase noise contributions with $S_{\phi}=0 \mathrm{~Hz}^{2} / \mathrm{Hz}$ (solid line) consistently predicts a sinusoidal dependence on position $y$. Theory assuming a phase noise level of $S_{\phi}=2 \pi \times 4 \mathrm{~Hz}^{2} / \mathrm{Hz}$ (half-solid line) accounts for phase noise and for the additional SNRP due to the trap (dotted line). The shaded area assumes a phase noise regime from half to twice the value of $S_{\phi}$.

decreases as expected with increasing power (solid line). This is in strong contrast to the low pressure regime, where measurements deviate from theoretical predictions, which yield a minimal temperature of $T_{\text {th }}=50 \mu \mathrm{K}$ at maximum input power $P_{\text {in }}=4 \mathrm{~mW}$ [Fig. 3(h)] [50].

In Fig. 4 we probe $T_{\text {c.m. }}$ versus particle position along the cavity axis $y$. We step the optical tweezers trap in increments of $\delta y=41 \mathrm{~nm}$ over a total distance exceeding $\lambda_{\text {cav }} / 2$. The cavity detuning is kept at a constant optimal value of $\Delta=-\Omega_{y}$ and at constant intracavity power $P_{\text {intra }}=1 \mathrm{~W}$. At high pressure [Fig. 4(a)] we observe a sinusoidal dependence of the temperature on position as expected from the optomechanical coupling strength $g_{0}$ [see Eq. (1)]. While the minimum temperature of $T_{\min }=$ $50 \mathrm{~K}$ agrees well with the theory (solid line), the maximum temperature differs by a factor of 2 from the expected room temperature of $T=295 \mathrm{~K}$. We attribute this to the particle motion at $T_{\text {c.m. }}=160 \mathrm{~K}$, which is $\delta y \approx 20 \mathrm{~nm}$ and thus a significant fraction of the intracavity standing wave $\lambda_{\text {cav }} / 2=532 \mathrm{~nm}$.

In the low pressure regime the situation is quite different [Figs. 4(b)-4(d)]. Periodic behavior is only observed for the lowest cooling powers $P_{\text {intra }}=5 \mathrm{~mW}$. Once the intracavity power is increased to $P_{\text {intra }}=20 \mathrm{~mW}, T_{\text {c.m. }}$. starts losing its position dependence. The minimum temperature $T_{\min } \approx$ $10 \mathrm{mK}$ persists over a broad region and loses its position dependence for $P_{\text {intra }}=172 \mathrm{~mW}$.

Altogether, as long as the dominant heating source is thermal noise, our observations are consistent with theory [see Eq. (2)]. Laser phase noise becomes significant below $p \leq 10^{-4}$ mbar preventing further cooling. The heating at low pressures cannot be explained by thermal heating (Fig. 2) or by photon radiation pressure (see Supplemental Material C).

Phase noise stems from a combination of cavity instability and phase noise of the driving laser. It translates into amplitude noise of the intracavity field. This has two effects on the system [35]: First, the optomechanical coupling strength $g$ changes due to its dependence on the intracavity photon number $n_{\text {cav }}$. However, for a laser linewidth of $\Gamma_{L}=$ $1 \mathrm{kHz}$ the coupling strength varies as $\kappa \Gamma_{L} / \Omega_{m}^{2} \ll 1$ and hence the dependence on intracavity field variations is negligible. Second, the conversion of phase to amplitude fluctuations inside the cavity gives rise to a stochastic force driving the mechanical oscillator. This leads to an additional phonon occupation $n_{\phi}=S_{\phi} n_{\text {cav }} / \kappa$, which scales linearly with the intracavity photon number $n_{\text {cav }}$ and the phase noise PSD at the mechanical frequency $S_{\phi}\left(\Omega_{m}\right)$. Including phase noise, the total final phonon occupation is

$$
n_{f}=n_{\mathrm{ph}}+n_{\phi}=\frac{\kappa^{2}}{16 \Omega_{m}^{2}}+\frac{\Gamma_{m} k_{B} T \kappa}{4 g_{0}^{2} n_{\mathrm{cav}} \hbar \Omega_{m}}+\frac{S_{\phi}}{\kappa} n_{\mathrm{cav}}
$$

where the first two terms derive from Eq. (2) and the last term accounts for phase noise. Equation (3) reproduces the data well (half-solid line), assuming the specified phase noise at $10 \mathrm{kHz}$ of $S_{\phi}=2 \pi \times 4 \mathrm{~Hz}^{2} / \mathrm{Hz}$. The shaded area covers a range of $S_{\phi} / 2$ and $2 \times S_{\phi}$ to account for the $1 / \Omega$ decrease in phase noise at higher frequencies [51] and additional phase noise contributions related to setup instabilities, respectively. In general, phase noise heating increases near the cavity resonance due to high intracavity photon numbers [see Eq. (3)] and dominates at low pressure. This leads to a shift in optimal detuning towards $\Delta<-\Omega_{m}$ and the opposite power dependence at high and low pressure. The trap SNRP is largely negligible [dotted line in Figs. 3(e)-3(h) and Eq. (C1) of Ref. [46]]. The optimum intracavity photon number $n_{\text {cav opt }}=\sqrt{\kappa^{2} \Gamma_{m} n_{\text {th }} /\left(4 g_{0}^{2} S_{\phi}\right)}$ depends on the phase noise level. Consequently, the minimum phonon occupation in presence of phase noise $S_{\phi}$ (see Supplemental Material C [46]) is 


$$
n_{f \text { min }}=n_{\text {min }}+\sqrt{\frac{S_{\phi} \Gamma_{m} n_{\text {th }}}{g_{0}^{2}}} .
$$

The experimental minimum phonon occupation of $n_{\mathrm{ph}}=$ 2100 , stands in good agreement with the theoretical prediction of $n_{f \min }=1750$, corresponding to $T_{\min }=10 \mathrm{mK}$ and $T_{f \min }=8.4 \mathrm{mK}$, respectively.

In conclusion, we experimentally and theoretically investigated the influence of phase noise heating in resolved sideband cooling of a levitated nanoparticle in high vacuum where thermal heating is no longer the main limitation. Counterintuitively, minimum temperatures are achieved at low intracavity power. Nevertheless, there are two approaches to continue towards GS cooling. Either the optomechanical coupling strength $g$ is increased by using a larger particle, a higher finesse, or a smaller cavity volume [52], such that the cooling efficiency per photon improves. Alternatively the coupling to the environment has to be reduced by further lowering the pressure or the system's phase noise [see Eq. (4)]. Reducing the current phase noise of $\sqrt{S_{\phi} /(2 \pi)}=2 \mathrm{~Hz} / \sqrt{\mathrm{Hz}}$ by a factor of 1500 , GS cooling can be achieved with the experimental parameters given here. This condition can be relaxed by an additional factor of 100 for a larger particle of $r=250 \mathrm{~nm}$ at a pressure of $P=10^{-10}$ mbar. Note that phase noise can be decreased with external filtering cavities acting as low pass filters [34,53]. This reduces the phase noise by several orders of magnitude [54], opening up the road to GS cooling with levitated nanoparticles.

N. M., A. D. L. R. S., P. M., and R. Q. acknowledge financial support from the European Research Council through Grant QnanoMECA (CoG-64790), Fundació Privada Cellex, CERCA Programme / Generalitat de Catalunya, and the Spanish Ministry of Economy and Competitiveness through the Severo Ochoa Programme for Centres of Excellence in R\&D Grants No. SEV-2015-0522 and No. FIS2016-80293-R. This project has received funding from the European Union's Horizon 2020 research and innovation programme under the Marie SkłodowskaCurie Grant Agreement No. 713729. J. G. received funding from the European Union's Marie Skłodowska-Curie program (SEQOO, H2020-MSCA-IF-2014, Grant No. 655369). L. N. and V. J. acknowledge support through the NCCR-QSIT program (Grant No. 51NF40-160591) by the Swiss National Science Foundation.

*nadine.meyer@icfo.eu

[1] J. Gieseler, L. Novotny, and R. Quidant, Nat. Phys. 9, 806 (2013).

[2] Y. Tsaturyan, A. Barg, A. Simonsen, L. G. Villanueva, S. Schmid, A. Schliesser, and E. S. Polzik, Opt. Express 22, 6810 (2014).
[3] C. Reinhardt, T. Müller, A. Bourassa, and J. C. Sankey, Phys. Rev. X 6, 021001 (2016).

[4] R. A. Norte, J. P. Moura, and S. Gröblacher, Phys. Rev. Lett. 116, 147202 (2016).

[5] A. H. Ghadimi, S. A. Fedorov, N. J. Engelsen, M. J. Bereyhi, R. Schilling, D. J. Wilson, and T. J. Kippenberg, Science 360, 764 (2018).

[6] W. Marshall, C. Simon, R. Penrose, and D. Bouwmeester, Phys. Rev. Lett. 91, 130401 (2003).

[7] D. Kleckner, I. Pikovski, E. Jeffrey, L. Ament, E. Eliel, J. van den Brink, and D. Bouwmeester, New J. Phys. 10, 095020 (2008).

[8] O. Romero-Isart, M. L. Juan, R. Quidant, and J. I. Cirac, New J. Phys. 12, 033015 (2010).

[9] O. Romero-Isart, A. C. Pflanzer, F. Blaser, R. Kaltenbaek, N. Kiesel, M. Aspelmeyer, and J. I. Cirac, Phys. Rev. Lett. 107, 020405 (2011).

[10] E. Hebestreit, M. Frimmer, R. Reimann, and L. Novotny, Phys. Rev. Lett. 121, 063602 (2018).

[11] Y. Arita, M. Mazilu, and K. Dholakia, Nat. Commun. 4, 2374 (2013).

[12] S. Kuhn, B. A. Stickler, A. Kosloff, F. Patolsky, K. Hornberger, M. Arndt, and J. Millen, Nat. Commun. 8, 1670 (2017).

[13] F. Monteiro, S. Ghosh, E. C. van Assendelft, and D. C. Moore, Phys. Rev. A 97, 051802(R) (2018).

[14] R. Reimann, M. Doderer, E. Hebestreit, R. Diehl, M. Frimmer, D. Windey, F. Tebbenjohanns, and L. Novotny, Phys. Rev. Lett. 121, 033602 (2018).

[15] L. Rondin, J. Gieseler, F. Ricci, R. Quidant, C. Dellago, and L. Novotny, Nat. Nanotechnol. 12, 1130 (2017).

[16] D. Hempston, J. Vovrosh, M. Toroš, G. Winstone, M. Rashid, and H. Ulbricht, Appl. Phys. Lett. 111, 133111 (2017).

[17] J. Gieseler and J. Millen, Entropy 20, 326 (2018).

[18] J. Bateman, S. Nimmrichter, K. Hornberger, and $H$. Ulbricht, Nat. Commun. 5, 4788 (2014).

[19] C. Wan, M. Scala, G. W. Morley, ATM. A. Rahman, H. Ulbricht, J. Bateman, P. F. Barker, S. Bose, and M. S. Kim, Phys. Rev. Lett. 117, 143003 (2016).

[20] J. Gieseler, B. Deutsch, R. Quidant, and L. Novotny, Phys. Rev. Lett. 109, 103603 (2012).

[21] V. Jain, J. Gieseler, C. Moritz, C. Dellago, R. Quidant, and L. Novotny, Phys. Rev. Lett. 116, 243601 (2016).

[22] F. Tebbenjohanns, M. Frimmer, A. Militaru, V. Jain, and L. Novotny, Phys. Rev. Lett. 122, 223601 (2019).

[23] G. P. Conangla, F. Ricci, M. T. Cuairan, A. W. Schell, N. Meyer, and R. Quidant, Phys. Rev. Lett. 122, 223602 (2019).

[24] T. Li, S. Kheifets, and M. G. Raizen, Nat. Phys. 7, 527 (2011).

[25] N. Kiesel, F. Blaser, U. Deli, D. Grass, R. Kaltenbaek, and M. Aspelmeyer, Proc. Natl. Acad. Sci. U.S.A. 110, 14180 (2013).

[26] P. Asenbaum, S. Kuhn, S. Nimmrichter, U. Sezer, and M. Arndt, Nat. Commun. 4, 2743 (2013).

[27] J. Millen, P. Z. G. Fonseca, T. Mavrogordatos, T. S. Monteiro, and P. F. Barker, Phys. Rev. Lett. 114, 123602 (2015).

[28] P. Z. G. Fonseca, E. B. Aranas, J. Millen, T. S. Monteiro, and P. F. Barker, Phys. Rev. Lett. 117, 173602 (2016). 
[29] V. Vuletić, H. W. Chan, and A. T. Black, Phys. Rev. A 64, 033405 (2001).

[30] C. Gonzalez-Ballestero, P. Maurer, D. Windey, L. Novotny, R. Reimann, and O. Romero-Isart, Phys. Rev. A 100, 013805 (2019).

[31] D. Windey, C. Gonzalez-Ballestero, P. Maurer, L. Novotny, O. Romero-Isart, and R. Reimann, Phys. Rev. Lett. 122, 123601 (2019).

[32] U. Delić, M. Reisenbauer, D. Grass, N. Kiesel, V. Vuletić, and M. Aspelmeyer, Phys. Rev. Lett. 122, 123602 (2019).

[33] A. Schliesser, R. Rivière, G. Anetsberger, O. Arcizet, and T. J. Kippenberg, Nat. Phys. 4, 415 (2008).

[34] A. H. Safavi-Naeini, J. Chan, J. T. Hill, S. Gröblacher, H. Miao, Y. Chen, M. Aspelmeyer, and O. Painter, New J. Phys. 15, 035007 (2013).

[35] P. Rabl, C. Genes, K. Hammerer, and M. Aspelmeyer, Phys. Rev. A 80, 063819 (2009).

[36] L. Diósi, Phys. Rev. A 78, 021801(R) (2008).

[37] R. Riedinger, A. Wallucks, I. Marinković, C. Löschnauer, M. Aspelmeyer, S. Hong, and S. Gröblacher, Nature (London) 556, 473 (2018).

[38] S. Hong, R. Riedinger, I. Marinković, A. Wallucks, S. G. Hofer, R. A. Norte, M. Aspelmeyer, and S. Gröblacher, Science 358, 203 (2017).

[39] R. Riedinger, S. Hong, R. A. Norte, J. A. Slater, J. Shang, A. G. Krause, V. Anant, M. Aspelmeyer, and S. Gröblacher, Nature (London) 530, 313 (2016).

[40] E. Verhagen, S. Deléglise, S. Weis, A. Schliesser, and T. J. Kippenberg, Nature (London) 482, 63 (2012).

[41] J. Chan, T. P. Alegre, A. H. Safavi-Naeini, J. T. Hill, A. Krause, S. Gröblacher, M. Aspelmeyer, and O. Painter, Nature (London) 478, 89 (2011).

[42] J. D. Teufel, T. Donner, D. Li, J. W. Harlow, M. S. Allman, K. Cicak, A. J. Sirois, J. D. Whittaker, K. W. Lehnert, and R. W. Simmonds, Nature (London) 475, 359 (2011).
[43] V. Vuletić and S. Chu, Phys. Rev. Lett. 84, 3787 (2000).

[44] D. R. Leibrandt, J. Labaziewicz, V. Vuletić, and I. L. Chuang, Phys. Rev. Lett. 103, 103001 (2009).

[45] M. Aspelmeyer, T. J. Kippenberg, and F. Marquardt, Rev. Mod. Phys. 86, 1391 (2014).

[46] See Supplemental Material at http://link.aps.org/supplemental/ 10.1103/PhysRevLett.123.153601 for the detailed derivation of the final steady state phonon occupation in the presence of laser phase noise and other omnipresent decoherence sources.

[47] V. Jain, Levitated optomechanics at the photon recoil limit, Doctoral dissertation, ETH, Zürich, Switzerland, 2017, https:// doi.org/https://dx.doi.org/10.3929/ethz-b-000200312.

[48] P. Mestres, J. Berthelot, M. Spasenović, J. Gieseler, L. Novotny, and R. Quidant, Appl. Phys. Lett. 107, 151102 (2015).

[49] E. Hebestreit, M. Frimmer, R. Reimann, C. Dellago, F. Ricci, and L. Novotny, Rev. Sci. Instrum. 89, 033111 (2018).

[50] The occupation number at $T_{\text {c.m. }}=50 \mu \mathrm{K}$ corresponds to $n_{\mathrm{ph}} \approx 8-10$. In a system with negligible laser phase noise heating $S_{\phi} \approx 0 \mathrm{~Hz}^{2} / \mathrm{Hz}$, GS cooling would be feasible by further lowering the heating rate (by decreasing the pressure $\mathrm{p}$ ) or improving the cooling rate $\Gamma_{\mathrm{opt}}$ (by increasing intracavity photon number $n_{\text {cav }}$ ) by 1 order of magnitude.

[51] K. Numata, A. W. Yu, J. B. Camp, and M. A. Krainak, Proc. SPIE Int. Soc. Opt. Eng. 10511, 49 (2018).

[52] L. Magrini, R. A. Norte, R. Riedinger, I. Marinković, D. Grass, U. Delić, S. Gröblacher, S. Hong, and M. Aspelmeyer, Optica 5, 1597 (2018).

[53] A. M. Jayich, J. C. Sankey, K. Børkje, D Lee, C Yang, M Underwood, L Childress, A Petrenko, S. M. Girvin, and J. G. E. Harris, New J. Phys. 14, 115018 (2012).

[54] J. Hald and V. Ruseva, J. Opt. Soc. Am. B 22, 2338 (2005). 